\title{
On Instability of LS-based Self-tuning Systems with Bounded Disturbances *
}

\author{
Shuai $\mathrm{Xu}^{\dagger}$, Chanying $\mathrm{Li}^{\dagger}$
}

January 9, 2019

\begin{abstract}
It is well known that discrete-time linear systems can be stabilized by a leastsquares (LS) based self-tuning regulator (STR), as long as noises are absent. However, this note shows that once the discrete-time linear systems are disturbed, the LS-based STR is always running the risk of unstabilizing systems, no matter how small the noises are.
\end{abstract}

Keywords: Least-squares, self-tuning regulators, parametrization, linear systems, discretetime, instability, noises

*The work was supported by the National Natural Science Foundation of China under Grants 61422308 and 11688101.

${ }^{\dagger} \mathrm{S} . \mathrm{Xu}$ and C. Li are with the Key Laboratory of Systems and Control, Academy of Mathematics and Systems Science, Chinese Academy of Sciences, Beijing 100190, P. R. China. They are also with the School of Mathematical Sciences, University of Chinese Academy of Sciences, Beijing 100049, P. R. China. Corresponding author: Chanying Li (Email: cyli@amss.ac.cn). 


\section{Introduction}

It was proved early in [1] that the following noise-free system

$$
A\left(q^{-1}\right) y_{t+1}=B\left(q^{-1}\right) u_{t}
$$

can be stabilized by a least-squares based self-tuning regulator. In fact, as long as the noise is absent, the LS-based STR even is capable of stabilizing the nonlinear discrete-time system

$$
y_{t+1}=\theta^{T} f_{t}\left(y_{t}, \ldots, y_{t-p+1}, u_{t-1}, \ldots, u_{t-q}\right),
$$

whenever $\left\|f_{t}(x)\right\|=O(1)+O\left(\|x\|^{b}\right)$ with $b<8$ (see [2]). But what will happen if systems are disturbed by bounded noises? This may be more practical. Relevant works in the stochastic framework shed some light (e.g., [3], [4], 5], 6], 7]). [7] studied the ARMA model, which is corrupted by a sequence of martingale difference noises, and derived the stability and optimality of the LS-based STR. Meanwhile, the strong consistency of the LS estimator is guaranteed in the closed loop. Later on, number $b=4$ and a polynomial criterion have been put forward as the critical nonlinear characterizations of the stabilizability for systems in type (11) but with random noises involved (see [8] and [9]). Such systems can be stabilized by the LS-based STR, when their nonlinearities are within the critical nonlinear conditions. Otherwise, no feedback control law is possible to stabilize them. This suggests that noises play an role here. The critical nonlinear growth rates are apparently reduced by the involvement of noises.

Now, a direct consequence of [6] indicates that if the noises are assumed to be bounded and i.i.d distributed, then with probability 1, the LS-based STR can stabilize system

$$
A\left(q^{-1}\right) y_{t+1}=B\left(q^{-1}\right) u_{t}+C\left(q^{-1}\right) w_{t+1}
$$

The trouble is, there still exist some sequences $\left\{w_{t}\right\}$ with probability 0 such that the stochastic tools could do nothing to them. [10] observed the divergence of the LS estimator in a self-tuning system for some special bounded noises. Nevertheless, whether the LS-based self-tuning system is stable or not for bounded noises was still unknown to people yet. We prove in this note 
that there indeed exist some bounded noises that will result in the instability of a LS-based selftuning system, even for the simplest discrete-time linear model with a scalar unknown parameter. Perhaps more surprisingly, as our result indicates, once a discrete-time system is disturbed, the LS-based STR is always running the risk of unstabilizing it, no matter how small the noises are. In the meantime, for the bounded noises causing the system unstable, the LS estimator is proved to be divergent during the closed-loop identification, as observed in [10].

Notably, though, discrete-time uncertain systems with bounded noises are stabilizable as well, provided their nonlinearities meet the polynomial criterion (see [11] and [12]). This means, different from the stochastic framework where the LS-STR converges to the minimal variance controller, the LS-STR in the deterministic framework performs no longer "optimal".

\section{$2 \quad$ Main Results}

Consider the discrete-time single-input/single-output linear model:

$$
y_{t+1}=\theta y_{t}+u_{t}+w_{t+1}, \quad t \geq 0
$$

where $y_{t}, u_{t}, w_{t} \in \mathbb{R}$ are the system output, input, and noise sequences, respectively. Parameter $\theta \in \mathbb{R}$ is unknown. Further, we assume

Assumption 1. There is a number $w>0$ such that $\left|w_{t}\right| \leq w$ for all $t \geq 1$.

The standard LS estimate $\theta_{t}$ of $\theta$ for model (2) reduces to

$$
\begin{aligned}
& \theta_{t+1}=\theta_{t}+\frac{1}{r_{t}} y_{t}\left(y_{t+1}-u_{t}-\theta_{t} y_{t}\right), \\
& \frac{1}{r_{t}}=\frac{1}{r_{t-1}}-\frac{y_{t}^{2}}{r_{t-1}^{2}+r_{t-1} y_{t}^{2}}, \quad r_{0}>0,
\end{aligned}
$$

where $\theta_{0}, r_{0}$ are the deterministic initial values of the algorithm. The feedback law is designed according to the well-known certainty equivalence principle:

$$
u_{t}=-\theta_{t} y_{t} .
$$


Denote $\tilde{\theta}_{t} \triangleq \theta-\theta_{t}$, then the closed-loop system (2) and (5) equals to

$$
y_{t+1}=\tilde{\theta}_{t} y_{t}+w_{t+1}
$$

Theorem 1. For any initial values $y_{0}$ and $\theta_{0}$, there exists a sequence $\left\{w_{t}\right\}$ satisfying Assumption 1 such that

(i) the LS estimate error $\tilde{\theta}_{t}$ diverges that $\varlimsup_{t \rightarrow+\infty}\left|\tilde{\theta}_{t}\right|=+\infty$;

(ii) the outputs of the closed-loop system (6) satisfies

$$
\varlimsup_{t \rightarrow+\infty} \frac{\sum_{i=1}^{t} y_{i}^{2}}{\sum_{i=1}^{t} w_{i}^{2}}=+\infty .
$$

\section{Proof of Theorem 1}

The proof is based on several lemmas.

Lemma 1. Let $k \geq 2$ be an integer that

$$
r_{k-1} \geq w^{2}, \quad\left|\tilde{\theta}_{k-1}\right| \leq 1, \quad\left|y_{k-1}\right| \leq \frac{w}{2 \sqrt{k-1}}
$$

Then, $\left|w_{t}\right| \leq w$ for all $t \geq k$, if

$$
w_{t}=-\tilde{\theta}_{t-1} y_{t-1}+\mathcal{S}\left(y_{t-1}\right) \frac{w}{2 \sqrt{t}}, \quad t \geq k
$$

where

$$
\mathcal{S}(x) \triangleq\left\{\begin{array}{ll}
-1, & \text { if } x<0 \\
1, & \text { if } x \geq 0
\end{array} .\right.
$$

Proof. First of all, note that (3) and (4) yield

$$
\tilde{\theta}_{t+1}=\tilde{\theta}_{t}-\frac{y_{t} y_{y+1}}{r_{t}}
$$

and $r_{t}=\sum_{i=1}^{t} y_{i}^{2}$ with $r_{0}=y_{0}^{2}$. According to (6) and (9), we have

$$
y_{t}=\mathcal{S}\left(y_{t-1}\right) \frac{w}{2 \sqrt{t}}, \quad t \geq k,
$$


We now verify that both $w_{k}$ and $w_{k+1}$ are bounded by $w$. As a matter of fact, by virtue of (6), (8) and (11),

$$
\begin{aligned}
\left|w_{k}\right| & \leq\left|\tilde{\theta}_{k-1} y_{k-1}\right|+\left|y_{k}\right| \\
& \leq \frac{w}{2}+\frac{w}{2}=w .
\end{aligned}
$$

Moreover, (10) yields

$$
\begin{aligned}
\left|\tilde{\theta}_{k}\right| & \leq\left|\tilde{\theta}_{k-1}\right|+\left|\frac{y_{k-1} y_{k}}{r_{k-1}}\right| \\
& \leq\left|\tilde{\theta}_{k-1}\right|+\left|\frac{y_{k-1} y_{k}}{w^{2}}\right| \\
& \leq 1+\frac{1}{4}=\frac{5}{4}
\end{aligned}
$$

which by (6) again,

$$
\left|w_{k+1}\right| \leq\left|\tilde{\theta}_{k} y_{k}\right|+\left|y_{k+1}\right| \leq \frac{5}{4} \frac{w}{2 \sqrt{2}}+\frac{w}{2} \leq w .
$$

When $t \geq k+1,\left|y_{t}\right| \leq\left|y_{t-1}\right|$ due to (11). In addition, (8) means

$$
r_{t-1} \geq r_{k-1} \geq w^{2}
$$

so, by (10) and (11),

$$
\begin{aligned}
\left|\tilde{\theta}_{t} y_{t}\right|-\left|\tilde{\theta}_{t-1} y_{t-1}\right| & \leq\left(\left|\tilde{\theta}_{t-1}\right|+\frac{y_{t-1} y_{t}}{r_{t-1}}\right)\left|y_{t}\right|-\left|\tilde{\theta}_{t-1} y_{t-1}\right| \\
& \leq\left|\frac{y_{t-1} y_{t}^{2}}{r_{t-1}}\right| \leq \frac{w^{3}}{8 r_{t-1} \sqrt{t-1} \sqrt{t} \sqrt{t}} \\
& \leq \frac{w}{8 \sqrt{t-1} \sqrt{t} \sqrt{t}}
\end{aligned}
$$

and

$$
\begin{aligned}
\left|y_{t}\right|-\left|y_{t+1}\right| & =\frac{w}{2}\left(\frac{1}{\sqrt{t}}-\frac{1}{\sqrt{t+1}}\right) \\
& =\frac{w}{2 \sqrt{t} \sqrt{t+1}(\sqrt{t}+\sqrt{t+1})} .
\end{aligned}
$$

If $t=2$, we have

$$
4 \sqrt{t-1} \sqrt{t}=4 \sqrt{2} \geq \sqrt{3}(\sqrt{2}+\sqrt{3})=\sqrt{t+1}(\sqrt{t}+\sqrt{t+1}) .
$$


For $t \geq 3$, it is also easy to compute

$$
\begin{aligned}
4 \sqrt{t-1} \sqrt{t} & =2 \sqrt{\frac{4}{3} t} \sqrt{3(t-1)} \\
& \geq 2 \sqrt{t+1} \sqrt{t+1} \\
& \geq \sqrt{t+1}(\sqrt{t}+\sqrt{t+1}) .
\end{aligned}
$$

Since integer $t \geq 2$, (13) and (14) shows

$$
\left|\tilde{\theta}_{t} y_{t}\right|-\left|\tilde{\theta}_{t-1} y_{t-1}\right| \leq\left|y_{t}\right|-\left|y_{t+1}\right|
$$

which yields

$$
\left|\tilde{\theta}_{t} y_{t}\right|+\left|y_{t+1}\right| \leq\left|\tilde{\theta}_{t-1} y_{t-1}\right|+\left|y_{t}\right|
$$

As a consequence, by (12), we have

$$
\begin{aligned}
\left|w_{t}\right| & =\left|y_{t}-\tilde{\theta}_{t-1} y_{t-1}\right| \leq\left|\tilde{\theta}_{t-1} y_{t-1}\right|+\left|y_{t}\right| \\
& \leq\left|\tilde{\theta}_{k} y_{k}\right|+\left|y_{k+1}\right|=w .
\end{aligned}
$$

The proof is completed.

Lemma 2. Let $k \geq 2$ be an integer fulfilling (8). If $\left\{w_{t}\right\}$ satisfies (9), then there is an integer $k^{\prime} \geq k$ such that $\left\{\left|\tilde{\theta}_{t}\right|, t \geq k^{\prime}\right\}$ is a strictly increasing sequence and $\lim _{t \rightarrow+\infty}\left|\tilde{\theta}_{t}\right|=+\infty$.

Proof. When $\left\{w_{t}\right\}$ satisfies (9), (111) holds and as $t \rightarrow+\infty$,

$$
r_{t+1}=r_{k}+\sum_{i=k+1}^{+\infty} y_{i}^{2}=r_{k}+\frac{w^{2}}{4} \sum_{i=k+1}^{+\infty} \frac{1}{i} \rightarrow+\infty .
$$

So, as $t \rightarrow+\infty$,

$$
\prod_{i=k+1}^{t}\left(1+\frac{y_{i+1}^{2}}{r_{i}}\right)=\prod_{i=k+1}^{t} \frac{r_{i+1}}{r_{i}}=\frac{r_{t+1}}{r_{k+1}} \rightarrow+\infty,
$$

which immediately shows

$$
\sum_{i=k+1}^{+\infty} \frac{y_{i+1}^{2}}{r_{i}}=+\infty
$$


Now, since $y_{t} y_{t+1} \geq 0$ for all $t \geq k+1$, by (11),

$$
\sum_{t=k+1}^{+\infty} \frac{y_{t} y_{t+1}}{r_{t}} \geq \sum_{t=k+1}^{+\infty} \frac{y_{t+1}^{2}}{r_{t}}=+\infty .
$$

In view of (10), $\tilde{\theta}_{t}$ decreases monotonically and $\lim _{t \rightarrow+\infty} \theta_{t}=-\infty$. Let $k^{\prime} \triangleq \min \left\{t: \tilde{\theta}_{t}<0\right\}$, then $\left\{\left|\tilde{\theta}_{t}\right|, t \geq k^{\prime}\right\}$ is a strictly increasing sequence.

Lemma 3. Let $j \geq 0$ be an integer that $y_{j} \neq 0, \tilde{\theta}_{j} \neq 0$ and $w_{t}=0$ for all $t \geq j+1$. The following two statements hold:

(i) there are some numbers $c_{0}>0, \alpha \in(0,1)$ and $t_{0} \geq j$ such that

$$
\left|y_{t}\right| \leq c_{0} \alpha^{t}, t \geq t_{0} \quad \text { and } \quad 0<\lim _{t \rightarrow+\infty}\left|\tilde{\theta}_{t}\right|<1
$$

(ii) if $\left|\tilde{\theta}_{j}\right| \geq 6$, then there is an integer $l \geq j$ such that

$$
r_{l-1} \leq 3 r_{j-1} \quad \text { and } \quad y_{l}^{2} \geq r_{j-1}
$$

Proof. We now prove statement (i). By (6) and (10),

$$
r_{t-1} \tilde{\theta}_{t}=r_{0} \tilde{\theta}_{1}-\sum_{i=1}^{t} y_{i-1} w_{i} .
$$

From (6), (10) and (16), for all $t \geq j$,

$$
\begin{aligned}
& y_{t+1}=\tilde{\theta}_{t} y_{t}, \\
& \tilde{\theta}_{t+1}=\frac{r_{t-1}}{r_{t}} \tilde{\theta}_{t}, \\
& r_{t-1} \tilde{\theta}_{t}=r_{j-1} \tilde{\theta}_{j} .
\end{aligned}
$$

Since $\left\{r_{t}\right\}$ is an increasing sequence, (18) shows that $\left\{\left|\tilde{\theta}_{t}\right|, t \geq j\right\}$ is a decreasing sequence. So, $\left|\tilde{\theta}_{t}\right|$ is bounded for all $t \geq j$ and $\lim _{t \rightarrow+\infty}\left|\tilde{\theta}_{t}\right|$ exists. We assert $\lim _{t \rightarrow+\infty}\left|\tilde{\theta}_{t}\right|<1$. Otherwise, 
if $\lim _{t \rightarrow+\infty}\left|\tilde{\theta}_{t}\right| \geq 1$, then $\left|\tilde{\theta}_{t}\right| \geq 1$ for all $j \geq t$. By (17), we have

$$
\begin{aligned}
r_{t} & =r_{j-1}+y_{j}^{2}+y_{j+1}^{2}+\cdots+y_{t}^{2} \\
& =r_{j-1}+y_{j}^{2}+\tilde{\theta}_{j}^{2} y_{j}^{2}+\cdots+\left(\prod_{i=j}^{t-1} \tilde{\theta}_{i}^{2}\right) y_{j}^{2} \\
& \geq r_{j-1}+(t-j+1) y_{j}^{2} .
\end{aligned}
$$

This immediately leads to $\lim _{t \rightarrow+\infty} r_{t}=+\infty$. By $\lim _{t \rightarrow+\infty}\left|\tilde{\theta}_{t}\right| \geq 1$ again,

$$
\lim _{t \rightarrow+\infty} r_{t-1} \tilde{\theta}_{t}=+\infty
$$

which contradicts to (19). Therefore, $\lim _{t \rightarrow+\infty}\left|\tilde{\theta}_{t}\right|<1$ and hence, $\left|\tilde{\theta}_{t_{0}}\right|<1$ for some integer $t_{0} \geq j$.

When $t \geq t_{0} \geq j$, by the fact that $\left\{\left|\tilde{\theta}_{t}\right|, t \geq j\right\}$ is a decreasing sequence, (17) shows that

$$
\left|y_{t}\right| \leq\left|y_{t_{0}}\right|\left|\tilde{\theta}_{t_{0}}\right|^{t-t_{0}}
$$

The first formula of (i) is thus derived by letting $c_{0}=\left|y_{t_{0}}\right|\left|\tilde{\theta}_{t_{0}}\right|^{-t_{0}}$ and $\alpha=\left|\tilde{\theta}_{t_{0}}\right| \in(0,1)$. So, as $t \rightarrow+\infty$,

$$
r_{t}=\sum_{i=1}^{t} y_{i}^{2}=O(1)+\sum_{i=t_{0}}^{t} c \alpha^{i}=O(1) .
$$

This together with (19) infers that $\lim _{t \rightarrow+\infty}\left|\tilde{\theta}_{t}\right|>0$.

To prove statement (ii), we first assert that there is an integer $l \geq j$ that $r_{l} \geq 3 r_{j-1}$. Otherwise, $r_{t-1}<3 r_{j-1}$ for all $t \geq j$. Therefore, by $\left|\tilde{\theta}_{j}\right| \geq 6$ and (19), we have for any $t \geq j$,

$$
\left|\tilde{\theta}_{t}\right|>\left|\frac{1}{3} \tilde{\theta}_{j}\right| \geq 2
$$

Consequently, by (20), as $t \rightarrow+\infty$,

$$
\begin{aligned}
r_{t} & =r_{j-1}+y_{j}^{2}+\tilde{\theta}_{j}^{2} y_{j}^{2}+\cdots+\left(\prod_{i=j}^{t-1} \tilde{\theta}_{i}^{2}\right) y_{j}^{2} \\
& >r_{j-1}+y_{j}^{2}+2 y_{j}^{2}+\cdots+2^{t-j} y_{j}^{2} \rightarrow+\infty,
\end{aligned}
$$


which contradicts to (21). Let

$$
l \triangleq \min \left\{t \geq j: r_{t} \geq 3 r_{j-1}\right\}
$$

then $r_{l-1} \leq 3 r_{j-1}$ and $r_{l} \geq 3 r_{j-1}$.

The remainder is devoted to showing $y_{l}^{2} \geq r_{j-1}$. Otherwise, if $y_{l}^{2}<r_{j-1}$, by noting that $r_{t-1} \leq 3 r_{j-1}$ for any $j+1 \leq t \leq l$, (22) holds as well for all $t \in[j+1, l]$ and hence $\frac{1}{\left|\tilde{\theta}_{t}\right|}<\frac{1}{2}$. Rewrite $r_{t}$ by

$$
\begin{aligned}
r_{l} & =r_{j-1}+y_{j}^{2}+\cdots+y_{l-1}^{2}+y_{l}^{2} \\
& =r_{j-1}+\frac{1}{\prod_{i=j}^{l-1} \tilde{\theta}_{i}^{2}} y_{l}^{2}+\cdots+\frac{1}{\tilde{\theta}_{l-1}^{2}} y_{l}^{2}+y_{l}^{2} \\
& <r_{j-1}+\frac{1}{2^{l-j}} y_{l}^{2}+\cdots+\frac{1}{2} y_{l}^{2}+y_{l}^{2}<3 r_{j-1},
\end{aligned}
$$

which derives a contradiction. (ii) is thus proved.

Lemma 4. Let $k \geq 2$ satisfy (8). Given a constant $c>0$, set

$$
w_{t}=\left\{\begin{array}{l}
-\tilde{\theta}_{t-1} y_{t-1}+\mathcal{S}\left(y_{t-1}\right) \frac{w}{2 \sqrt{t}}, \quad k \leq t \leq j \\
0, \quad t \geq j+1
\end{array}\right.
$$

where $j \geq k$ is an integer such that

$$
\left|\tilde{\theta}_{j}\right| \geq \max \left\{\left|\tilde{\theta}_{0}\right|,\left|\tilde{\theta}_{1}\right|, \ldots,\left|\tilde{\theta}_{j-1}\right|, 6,126 c\right\} .
$$

Then, $\left(\sum_{i=1}^{+\infty} y_{i}^{2}\right) /\left(\sum_{i=1}^{+\infty} w_{i}^{2}\right) \geq c$.

Proof. By (6), (8), (23) and (24), we have

$$
\begin{aligned}
\sum_{i=1}^{+\infty} w_{i}^{2} & =\sum_{i=1}^{j} w_{i}^{2}=\sum_{i=1}^{j}\left(y_{i}-\tilde{\theta}_{i-1} y_{i-1}\right)^{2} \\
& \leq 2 \sum_{i=1}^{j} y_{i}^{2}+2 \sum_{i=1}^{j} \tilde{\theta}_{i-1}^{2} y_{i-1}^{2} \\
& \leq 2\left(r_{j-1}+y_{j}^{2}\right)+2 r_{j-1} \tilde{\theta}_{j}^{2} \\
& \leq 2 r_{j-1}+2\left(2 y_{j-1}^{2} \tilde{\theta}_{j-1}^{2}+2 w^{2}\right)+2 r_{j-1} \tilde{\theta}_{j}^{2} \\
& \leq 2 r_{j-1}+4 w^{2}+4 r_{j-1} \tilde{\theta}_{j}^{2}+2 r_{j-1} \tilde{\theta}_{j}^{2} \leq 7 r_{j-1} \tilde{\theta}_{j}^{2} .
\end{aligned}
$$


Note that $w_{t}=0$ for all $t \geq j+1$, in view of Lemma 3, there exists an integer $l \geq j$ fulfilling (15). By (19), $r_{l-1} \leq 3 r_{j-1}$ yields $\left|\tilde{\theta}_{l}\right|^{2} \geq \frac{1}{9}\left|\tilde{\theta}_{j}\right|^{2}$. Then, (15), (17) and (19) imply

$$
\begin{aligned}
y_{l+1}^{2}+y_{l+2}^{2} & =\tilde{\theta}_{l}^{2} y_{l}^{2}\left(1+\tilde{\theta}_{l+1}^{2}\right) \geq 2 \tilde{\theta}_{l}^{2} y_{l}^{2}\left|\tilde{\theta}_{l+1}\right| \\
& \geq \frac{2}{9} \tilde{\theta}_{j}^{2} y_{l}^{2} \frac{r_{j-1}\left|\tilde{\theta}_{j}\right|}{r_{l-1}+y_{l}^{2}} \geq \frac{2}{9} r_{j-1}\left|\tilde{\theta}_{j}\right|^{3} \frac{y_{l}^{2}}{3 r_{j-1}+y_{l}^{2}} \\
& \geq \frac{1}{18} r_{j-1}\left|\tilde{\theta}_{j}\right|^{3} .
\end{aligned}
$$

Moreover, $\left|\tilde{\theta}_{j}\right| \geq 126 c$, the above inequality shows

$$
\frac{\sum_{i=1}^{+\infty} y_{i}^{2}}{\sum_{i=1}^{+\infty} w_{i}^{2}} \geq \frac{y_{l+1}^{2}+y_{l+2}^{2}}{7 r_{j-1} \tilde{\theta}_{j}^{2}} \geq c
$$

which completes the proof.

Proof of Theorem 1. Set the noise

$$
w_{t}=\left\{\begin{array}{ll}
\mathcal{S}\left(\tilde{\theta}_{0} y_{0}\right) w, & t=1 \\
0, & 2 \leq t \leq k_{1}-1
\end{array},\right.
$$

where for $\mathcal{K}_{1} \triangleq\{k \geq 3:(8)$ holds $\}$,

$$
k_{1} \triangleq\left\{\begin{array}{ll}
\min _{k \in \mathcal{K}_{1}} k, & \mathcal{K}_{1} \neq \emptyset \\
+\infty, & \mathcal{K}_{1}=\emptyset
\end{array} .\right.
$$

Clearly, $\left|w_{t}\right| \leq w$ for $t \in\left[1, k_{1}-1\right]$. We next claim that $k_{1}$ is finite. Otherwise, $\mathcal{K}_{1}=\emptyset$. Then, (8) fails for every $k \geq 3$ and $w_{t}=0$ whenever $t \geq 2$. Now, $w_{1}=\mathcal{S}\left(\tilde{\theta}_{0} y_{0}\right) w$, which means $\left|w_{1}\right|=w$ and $y_{1}^{2} \geq w^{2}>0$. Therefore,

$$
r_{k-1} \geq r_{1} \geq w^{2}, \quad \forall k \geq 3
$$

If $\tilde{\theta}_{1}=0$, it is easy to compute that $\tilde{\theta}_{2}=y_{2}=0$ due to $w_{2}=0$. So, (8) holds for $k=3$. This asserts $\tilde{\theta}_{1} \neq 0$. Consequently, by Lemma $3(\mathrm{i})$, there are some $c_{0}>0$ and $\alpha \in(0,1)$ such that for all sufficiently large $k \geq 3$,

$$
\left|y_{k-1}\right| \leq c_{0} \alpha^{k-1} \leq \frac{w}{2 \sqrt{k-1}} \quad \text { and } \quad\left|\tilde{\theta}_{k-1}\right|<1
$$


which together with (26) contradicts to $\mathcal{K}_{1}=\emptyset$. Therefore, $k_{1}$ is finite.

Now, fix $k_{1} \in \mathcal{K}_{1}$. Define

$$
j_{1} \triangleq\left\{\begin{array}{ll}
\min _{j \in \mathcal{J}_{1}} j, & \mathcal{J}_{1} \neq \varnothing \\
+\infty, & \mathcal{J}_{1}=\varnothing
\end{array},\right.
$$

where

$$
\mathcal{J}_{1} \triangleq\left\{j \geq k_{1}:\left|\tilde{\theta}_{j}\right| \geq \max \left\{\left|\tilde{\theta}_{0}\right|,\left|\tilde{\theta}_{1}\right|, \ldots,\left|\tilde{\theta}_{j-1}\right|, 126\right\}\right\}
$$

Moreover, let

$$
k_{2} \triangleq \begin{cases}\min _{k \in \mathcal{K}_{2}} k, & \mathcal{K}_{2} \neq \varnothing \\ +\infty, & \mathcal{K}_{2}=\varnothing\end{cases}
$$

with

$$
\mathcal{K}_{2} \triangleq\left\{k \geq j_{1}+2: \text { (8) holds and } \sum_{i=1}^{k-1} y_{i}^{2} \geq \sum_{i=1}^{k-1} w_{i}^{2}\right\} \text {. }
$$

For $j_{1}$ and $k_{2}$ defined above, set

$$
w_{t}=\left\{\begin{array}{ll}
-\tilde{\theta}_{t-1} y_{t-1}+\mathcal{S}\left(y_{t-1}\right) \frac{w}{2 \sqrt{t}}, & k_{1} \leq t \leq j_{1} \\
0, & j_{1}+1 \leq t \leq k_{2}-1
\end{array} .\right.
$$

Noting that (8) is true for $k=k_{1}$, by Lemma 1, $\left|w_{t}\right| \leq w$ for all $t \in\left[k_{1}, k_{2}-1\right]$.

We proceed to prove that both $j_{1}$ and $k_{2}$ are finite. If $j_{1}=+\infty$, then $w_{t}$ satisfies (9) for all $t \geq k_{1}$. Further, since (8) holds for $k=k_{1}$, by Lemma $2,\left\{\left|\tilde{\theta}_{t}\right|, t \geq k_{1}^{\prime}\right\}$ is an increasing sequence for some $k_{1}^{\prime} \geq k_{1}$ and $\lim _{t \rightarrow+\infty}\left|\tilde{\theta}_{t}\right|=+\infty$, which gives $\mathcal{J}_{1} \neq \emptyset$. Hence $j_{1}$ is finite or a contradiction arises. So, by Lemma \&, we immediately deduce that for all sufficiently large $k$,

$$
\sum_{i=1}^{k-1} y_{i}^{2} \geq \sum_{i=1}^{k-1} w_{i}^{2}
$$

It is clear that $\tilde{\theta}_{j_{1}} \neq 0$ as $j_{1} \in \mathcal{J}_{1}$ and $y_{j_{1}} \neq 0$ by (11). Similar to the proof of $k_{1}<+\infty$, Lemma 3(i) shows that $k_{2}$ is finite.

Suppose two increasing sequences $\left\{k_{i} \in \mathbb{N}^{+}, 1 \leq i \leq s\right\},\left\{j_{i} \in\left[k_{i}, k_{i+1}-2\right] \cap \mathbb{N}^{+}, 1 \leq i \leq s-1\right\}$ and a series $\left\{w_{t}, 1 \leq t \leq k_{s}-1\right\}$ are constructed for some $s \geq 2$ such that (으) holds for $k=k_{s}$, $\left|\tilde{\theta}_{j_{s-1}}\right| \geq 126(s-1)$ and

$$
\sum_{i=1}^{k_{s}-1} y_{i}^{2} \geq(s-1) \sum_{i=1}^{k_{s}-1} w_{i}^{2}
$$


Analogous arguments of (27) and (28)-(29) yield that there are two finite integers $k_{s+1}$ and $j_{s}$, as well as a sequence $\left\{w_{t}, k_{s} \leq t \leq k_{s+1}-1\right\}$ such that (8) holds for $k=k_{s+1},\left|\tilde{\theta}_{j_{s}}\right| \geq 126 s$ and

$$
\sum_{i=1}^{k_{s+1}-1} y_{i}^{2} \geq s \sum_{i=1}^{k_{s+1}-1} w_{i}^{2} .
$$

So, there exists a $\left\{w_{t}, t \geq 1\right\}$ and a $\left\{\left(k_{s}, j_{s}\right), s \geq 1\right\}$ fulfilling (30) and $\left|\tilde{\theta}_{j_{s}}\right| \geq 126 s$ for each $s \geq 2$. Statements (i) and (ii) are thus derived as desired.

\section{References}

[1] G. Goodwin, P. Ramadge, P. Caines, Discrete-time multivariable adaptive control, IEEE Transactions on Automatic Control 25 (3) (1980) 449-456.

[2] L. Guo, C. Wei, Global stability/instability of LS-based discrete-time adaptive nonlinear control, IFAC Proceedings Volumes 29 (1) (1996) 5215-5220.

[3] K. J. Åström, B. Wittenmark, On self tuning regulators, Automatica 9 (2) (1973) 185-199.

[4] K. J. Åström, U. Borisson, L. Ljung, B. Wittenmark, Theory and applications of self-tuning regulators, Automatica 13 (5) (1977) 457-476.

[5] P. Kumar, Convergence of adaptive control schemes using least-squares parameter estimates, IEEE Transactions on Automatic Control 35 (4) (1990) 416-424.

[6] L. Guo, H.-F. Chen, The Åström-Wittenmark self-tuning regulator revisited and ELS-based adaptive trackers, IEEE Transactions on Automatic Control 36 (7) (1991) 802-812.

[7] L. Guo, Convergence and logarithm laws of self-tuning regulators, Automatica 31 (3) (1995) 435-450.

[8] L. Guo, On critical stability of discrete-time adaptive nonlinear control, IEEE Transactions on Automatic Control 42 (11) (1997) 1488-1499. 
[9] C. Li, J. Lam, Stabilization of discrete-time nonlinear uncertain systems by feedback based on LS algorithm, SIAM Journal on Control and Optimization 51 (2) (2013) 1128-1151.

[10] S. V. Gusev, Gradient estimation algorithm with dead zone for adaptive control of discretetime plants with mean square bounded disturbances, in: Control Conference (ECC), European, IEEE, 1993, pp. 2222-2225.

[11] C. Li, L.-L. Xie, On robust stability of discrete-time adaptive nonlinear control, Systems \& control letters 55 (6) (2006) 452-458.

[12] C. Li, L.-L. Xie, L. Guo, A polynomial criterion for adaptive stabilizability of discrete-time nonlinear systems, Communications in Information \& Systems 6 (4) (2006) 273-298. 